Vol.15, No. 55, April, 2020, 560-572

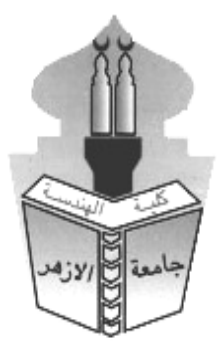

\title{
ORBIT CONTROL USING ELECTRIC PROPULSION SYSTEMS FOR EARTH OBSERVATION SATELLITE SWARM
}

\author{
Tarek Mahmoud*1, Saed Othman ${ }^{1}$, A. Farrag ${ }^{2}$ and Ahmed Y. ELRaffiey ${ }^{2}$ \\ ${ }^{1}$ Electrical Eng. Department Faculty of Engineering, Al-Azhar University, Cairo, Egypt \\ ${ }^{2}$ Division Egyptian Space Agency, Cairo, ,Egypt \\ * Corresponding Author E-mail: tarek.naser.2014@azhar.edu.eg
}

\begin{abstract}
The new trend in a remote sensing application is to replace the traditional single satellite with a set of low-cost, simple and short development time satellites. Satellite swarm consists of twelve CubeSats work as imaging nodes accompanied by a data-relay-satellite was proposed to fulfill Earth observation mission. In order to achieve the required mission performance, it is necessary to maintain the virtual structure of the satellite swarm over the mission lifetime. The external disturbances (e.g. Earth oblateness, atmospheric drag, and solar radiation pressure, etc.) cause a drift to the orbits of satellite warm; therefore, this disturbance can change the virtual topology of the satellite swarm. In order to keep the virtual topology of the satellite swarm, and to fulfill the mission requirements, it is required to maintain the orbits of all satellite swarm members all over its lifetime. Many researchers studied autonomous orbit control algorithms, such as PD, LQR, and sliding mode. In order to select the best algorithm for satellite swarm mission, this paper makes a comparison between the commonly used orbit control algorithms based on Matlab simulation, the required control thrust is generated using electric propulsion system, The parameters of control performance such as; the settling time, the steady-state error, the required control effort, and the execution time are used as compression factors to select the suitable orbital control algorithm for the proposed satellite swarm.
\end{abstract}

\section{KEYWORDS: Satellite Swarm, Earth Oblateness, Virtual Topology, Autonomous Orbit, Electric} Propulsion

$$
\begin{aligned}
& \text { التحمم في المدارات باستخدام أنظمة الدفع الكهربائية لسرب أقمار صناعية فى مراقبة الأرض }
\end{aligned}
$$

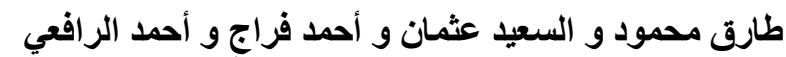

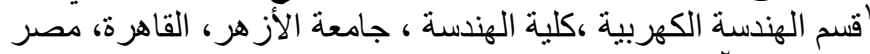

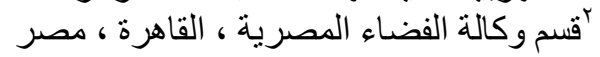

$$
\begin{aligned}
& \text { E-mail: tarek.naser.2014@azhar.edu.eg البريد الإليكتروني للباحث الرئيسي_ وكة الفئ }
\end{aligned}
$$

الاتجاه الجديد في تطبيق الاستشعار عن بعد هو استبدال القمر الصناعي التقليدي بمجموعة من الأقمار الصناعية ذات التكلفة

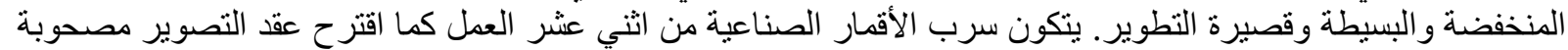

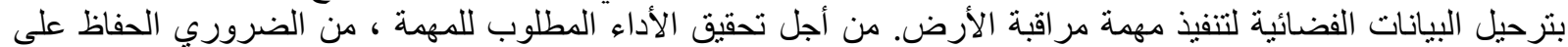

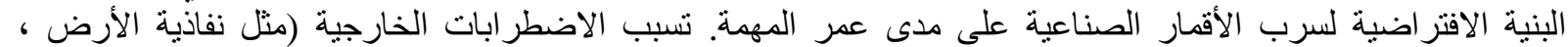

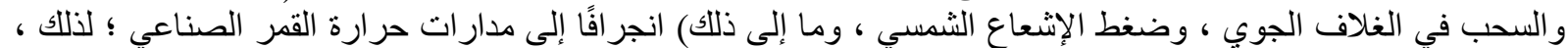

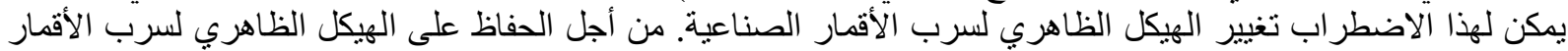

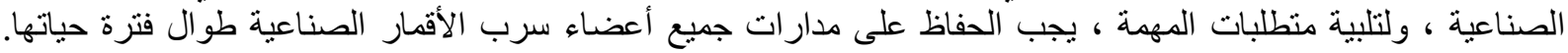




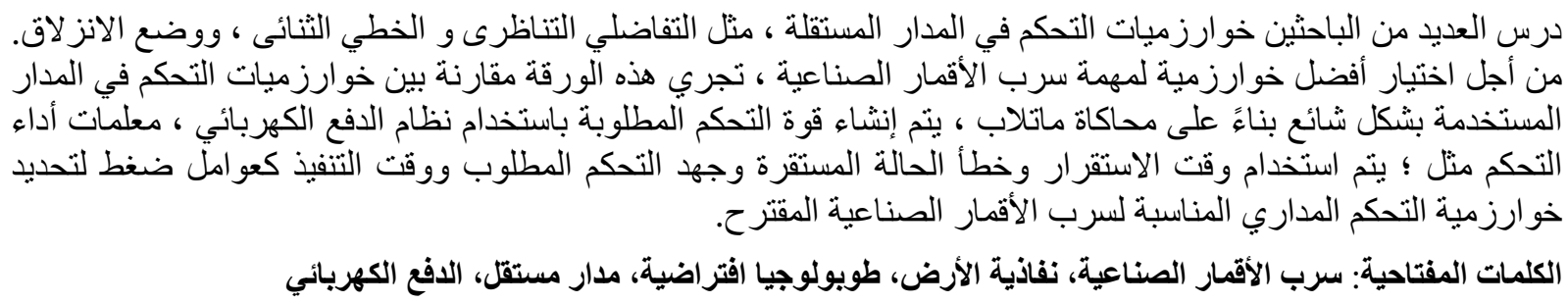

\section{SYMBOLS}

\begin{tabular}{|c|c|}
\hline$r$ & Vector of satellite position \\
\hline$y$ & Vector of satellite velocity \\
\hline$a g$ & Vector of acceleration due to Earth gravity \\
\hline$a c$ & Vector of the control thrust \\
\hline$a d$ & $\begin{array}{l}\text { Vector of total acceleration due } \\
\text { perturbation }\end{array}$ \\
\hline$a_{\text {aeri }}$ & $\begin{array}{l}\text { Vector of total acceleration due } \\
\text { aerodynamic force }\end{array}$ \\
\hline$a_{j 2}$ & Vector of total acceleration due to $\mathrm{J} 2$ effect \\
\hline$a_{m g}$ & $\begin{array}{l}\text { Vector of total acceleration due to the earth } \\
\text { magnetic }\end{array}$ \\
\hline$a_{\text {swn }}$ & $\begin{array}{l}\text { Vector of total acceleration due to sun } \\
\text { pressure }\end{array}$ \\
\hline$r_{r e f}$ & Vector of reference satellite position \\
\hline$v_{r a f}$ & Vector of reference satellite velocity \\
\hline & of acceleration due to Earth gravity \\
\hline
\end{tabular}

$\begin{array}{lll} & \text { at the reference position } \\ \boldsymbol{\Delta} r & : & \text { Vector of error in satellite position } \\ \boldsymbol{\Delta} v & : \text { Vector of error in satellite velocity } \\ u & : & \text { Vector of required control effort } \\ K_{p} & : & \text { The proportional gain of the PD controller } \\ K_{d} & : & \text { The derivative gain of the PD controller } \\ \omega_{n} & : \text { Natural frequency } \\ \zeta & : \text { Damping ratio } \\ I & : \text { performance index } \\ K & : \text { The LQR gain } \\ S & : \text { The sliding manifold of the sliding mode } \\ & \quad \text { control } \\ K_{1}, K: & \text { Gains of the sliding mode control } \\ V & : \text { Lyapunov candidate function }\end{array}$

\section{INTRODUCTION}

Satellite swarm is considered as multi-satellite mission or distributed space systems, it consists of three or more satellites flying in adjacent orbits, they form a virtual structure according to their mission For remote seining applications, this satellite swarm can provide an image of a ground target from different angles at the same time (If all satellites in the swarm imaged the same target at the same time), or it can increase the swath width of imaged area (If all satellites in the swarm imaged adjacent ground areas). The concept of satellite swarm is still a new concept and only a few missions were launched and successfully demonstrated the swarm concept, such as European space agency (ESA) Swarm for Earth magnetic field monitoring and radio telescope based on Nano-satellites in moon orbit called OLFAR mission

A Family Swarm concept for remote sensing application was proposed in, it consists of eleven CubeSat and one micro-satellite working as imaging nodes, besides one data relay satellite, the imaging nodes are used to image the ground targets, while, the data relay satellite is used to collect the imaging data from the imaging nodes and transfer it to the ground station.

The external disturbances (e.g. Earth oblations, atmospheric drag, and solar radiation pressure, etc.) cause a drift to the orbits thus, this disturbance can change the virtual topology of the satellite swarm. In order to keep the virtual topology of the satellite swarm, and to fulfill the mission requirements it is required to maintain the orbits of all Family satellite swarm members, this maintenance should cover the swarm lifetime. The orbit maintenance problem means a series of orbit correction, this correction is fulfilled by the propulsion sub-system. The orbit correction can be achieved using commands from the ground, or it is fulfilled autonomously by onboard control algorithms, the autonomous onboard orbit control is well established and used in many missions], where it can perform a full orbit control with high accuracy, real-time, and with a significant reduction in ground operations. 
In order to increase the control accuracy, the maneuver cycle must be reduced; consequently, the choice of using an autonomous orbit control system will be the best choice. The reduced size of the maneuvers in autonomous orbit control allows the use of a low thrust propulsion system (such as an electric propulsion sub-system) is usedThe electric propulsion subsystems are characterized by their simplest and compact size

Many researchers studied autonomous orbit control algorithms, such as PD, LQR, and sliding mode. In this paper, a comparison between the commonly used orbit control algorithm for a satellite swarm is conducted, this comparison is used to select the suitable orbital control algorithm for a family satellite swarm. For this purpose, the paper is organized as follows; the mission of satellite family swarm is presented in section two, orbital dynamics is driven in section three, the commonly used control algorithms are studied in section four, section five is dedicated for the simulation results, and the conclusion of the research is presented in section six.

\section{THE MISSION OF SATELLITE FAMILY SWARM}

The mission of this satellite swarm is to image any target in the Earth within less than 7 days with resolution better than $2.5 \mathrm{~m}$, and swath width $=240 \mathrm{~km}$ (all satellites in the swarm imaged adjacent ground areas), or, with resolution better than $0.5 \mathrm{~m}$ with swath width $20 \mathrm{~km}$ (when all satellites in the swarm imaged the same target at the same time), moreover, the ground station can send imaging commands and receive the telemetry and image data every orbital period (i.e. less than $100 \mathrm{~min}$ ).

This satellite swarm consists of eleven CubeSats and one Micro-satellite working as imaging nodes, besides a data relay satellite, the imaging nodes collect the earth images and send them to the data-relay-satellite, which by its turn will resend these imaging data to the ground station

The satellite swarm can be seen as Family Swarm, where the data-relay-satellite is acting as a father satellite (F-Sat.) and Micro-satellite is acting as a mother Satellite (M-Sat.), which fly with its imaging nodes or children satellites (C-Sat.).

\subsection{Functions of family swarm members}

The functions of each member of the family swarm are as follows.

\subsubsection{The function of F-Sat.}

F-Sat communicates with the ground station one session per orbit to receive the commands of all swarm members and send all imaging and telemetry data which are collected from swarm members to the ground station .

F-Sat communicates with the M-sat two sessions per orbit in-order to resend the received commands (i.e. commands for $\mathrm{M}$-Sat and $\mathrm{C}-\mathrm{Sat}$ ) and receive the imaging data and Telemetry (i.e. image and telemetry data collected by all C-Sats and M-Sats )

\subsubsection{Function of M-Sat}

Communicate with each C-sat one session per orbit to resend the received commands and Collect the telemetry and imaging data from the corresponding C-Sat,Execute its imaging commands

\subsubsection{Function of C-Sat}

Communicate with M-Sat one session per orbit to receive imaging commands and send imaging and Telemetry data to M-Sat,Execute the imaging commands.

\subsection{Orbit requirements}

In order to fulfill the required mission, the following orbital requirements should be maintained all over the lifetime of the satellite swarm:-

1) Imaging nodes are orbiting the Earth in four sun-synchronous orbits, with $490 \mathrm{Km}$ altitude and, $97.37^{\circ}$ inclination angle.

2) The distance between each adjacent imaging nodes should be $4 \mathrm{~km}$.

3) F-Sat flays in an equatorial orbit with an altitude of $497 \mathrm{~km}$ in. 
4) Each member in the swarm should maintain its orbit within accuracy not worse than $0.15 \mathrm{~km}$ in the of its position and $1 \frac{\mathrm{m}}{\mathrm{s}}$ in the of its velocity

\section{System dynamic model}

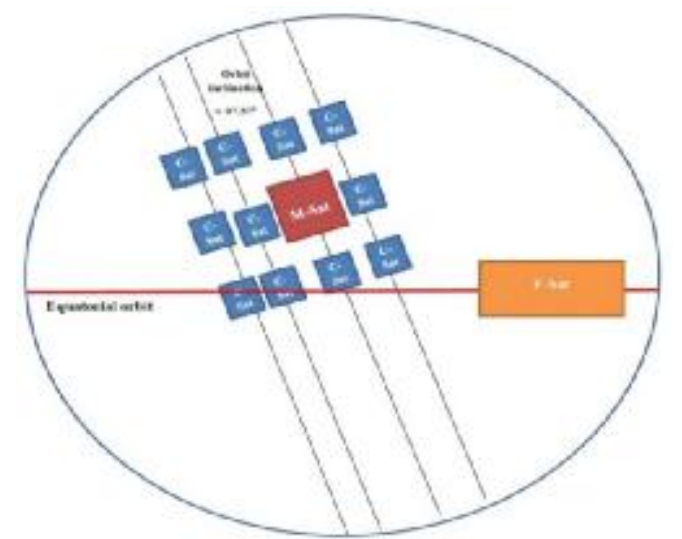

The dynamic model that describes satellite position and velocity in the inertial coordinate system (ICS) ]is described as.

$$
\begin{aligned}
& \dot{\mathbf{r}}=\mathrm{v} \\
& \dot{\mathbf{v}}=-\mathrm{ag}+\mathrm{ac}+\mathrm{ad} \\
& \mathrm{ad}=\mathrm{a}_{\mathrm{aero}}+\mathrm{a}_{\mathrm{j} 2}+\mathrm{a}_{\mathrm{mg}}+\mathrm{a}_{\mathrm{sun}}
\end{aligned}
$$

Satellite position and velocity control loop is shown in Figure 2, where, where the actual position and velocity, $r, v$ are compared with the reference position and velocity $r_{r e f}, v_{r e f}$, then the error in position and velocity $\Delta r, \Delta v$ are used by the orbit controller to generate the required acceleration, ac (i.e. corrective action) to track the reference values and compensate effects of disturbances ad.

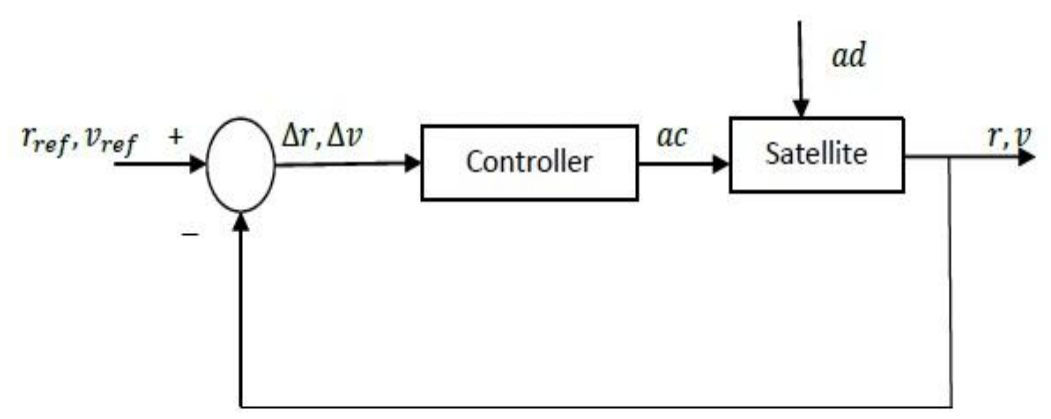

Figure 2 , Satellite position and velocity control loop

\section{Family Swarm orbit maintenance}

In order to force the satellite to track a reference orbit, many orbital maneuvers should be done, these maneuvers are performed using propellant. The less consumption of propellant is needs, the 
lighter the satellite can stay in orbit and the less the mission will cost. Therefore, the amount of consumed propellant is a crucial factor in designing the mission.

Maintaining a reference orbit can be obtained by single or multiple impulses, however, the low accurate orbital maneuver can be achieved by a single impulse, but any desired orbital maneuver can be achieved with high accuracy by multiple impulses .

\section{CONTROL CONCEPT}

The reference orbit of each member (i.e. reference of satellite position and velocity) in this work will be considered as an ideal orbit and it will be calculated from solving the two-body problem without any perturbations as described in the system ( 4 ) \&( 5).

$$
\begin{aligned}
& \dot{\mathbf{r}}_{\mathrm{ref}}=\mathbf{v}_{\mathrm{ref}} \\
& \dot{\mathbf{v}}_{\mathrm{ref}}=-\mathrm{a} \mathbf{\Xi}_{\mathrm{ref}}
\end{aligned}
$$

Each member in the family swarm must maintain its reference orbit in order to fulfill the mission requirements described in section (2.2), with minimum settling time, minimum exaction time and minimum control effort using thrusters in $\mathrm{x}, \mathrm{y}$, and z-axis. A control action will be taken by the controller if the error of the satellite position exceeds $0.5 \mathrm{~kg}$ and/or the error of its velocity exceeds $5 \frac{m}{s}$

The system of errors in position and velocity after neglecting the disturbance term can be described as follows

$$
\begin{aligned}
& \Delta \mathrm{r}=\mathrm{r}_{\text {ref }}-\mathrm{r} \\
& \Delta \mathrm{v}=\mathrm{v}_{\mathrm{ref}}-\mathrm{v} \\
& \dot{\Delta \mathrm{r}}=\dot{\mathrm{r}}_{\mathrm{ref}}-\dot{\mathrm{r}}=\mathrm{v}_{\mathrm{ref}}-\mathrm{v} \\
& \dot{\Delta \mathrm{r}}=\Delta \mathrm{v} \\
& \dot{\Delta v}=\dot{\mathrm{v}}_{\mathrm{ref}}-\dot{\mathrm{v}} \\
& \dot{\Delta v}=-\mathrm{ag}_{\mathrm{ref}}+\mathrm{ag}-\mathrm{ac}
\end{aligned}
$$

Hence, the state-space model which represents the system (9)\&(11) can be considered as follows

$$
\dot{x}=\mathbf{A} x+\mathbf{B u}
$$

Where

$$
\begin{aligned}
& \mathbf{x}=\left[\begin{array}{l}
\Delta \mathrm{r} \\
\mathbf{\Delta I}
\end{array}\right] \\
& \mathbf{A}=\left[\begin{array}{ll}
\mathbf{0} & \mathbf{1} \\
0 & 0
\end{array}\right] \& \quad B=\left[\begin{array}{l}
0 \\
1
\end{array}\right] \\
& \mathbf{u}=-a g_{\text {ref }}+a g-a c
\end{aligned}
$$

The difference between the magnitude of actual orbital position/velocity described in equations ( $1) \&(2) \&(3)$ and the magnitude of reference orbital position/velocity described in equations ( $4) \&(5)$ within 50 orbital periods are shown in Figure 3, and Figure 4

As it is shown in

Figure 3, Figure 4, the external disturbances will force the actual orbital parameters $r, v$ to drift away from the reference parameters $r_{\text {ref }}, v_{\text {ref }}$; consequently, an 
orbit control algorithm is required to maintain the reference orbital parameters and neglect the effect of external disturbances.

In the next section, a brief description for the commonly used algorithms in orbit control process are presented, and, a comparison between these algorithms will be used to choose the best orbit control technique for the family swarm, the comparison factors which are used to judge the performance of these algorithms according to their priority are below

1) Minimum required control thrust.

2) Achievement of Steady stat error; less than $0.15 \mathrm{~km}$ in position and $1 \frac{\mathrm{m}}{\mathrm{s}}$ in velocity.

3) Minimum settling time (i.e. the required time to reach $95 \%$ of the reference value).

4) Minimum execution time (i.e. time needed by processor to fulfill the controller task).

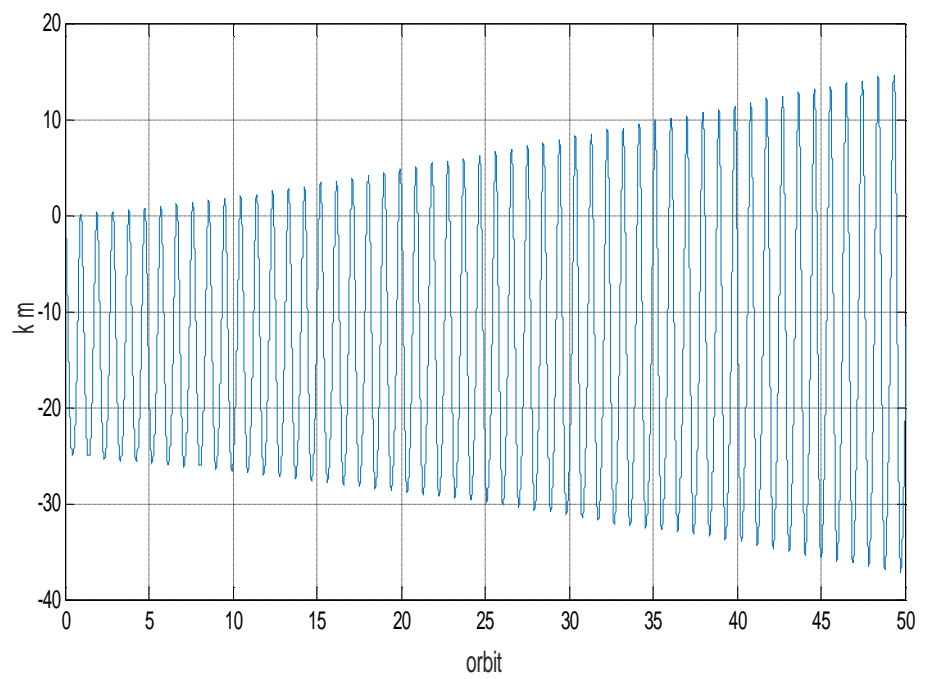

Figure 3, the magnitude of error in orbit position magnitude

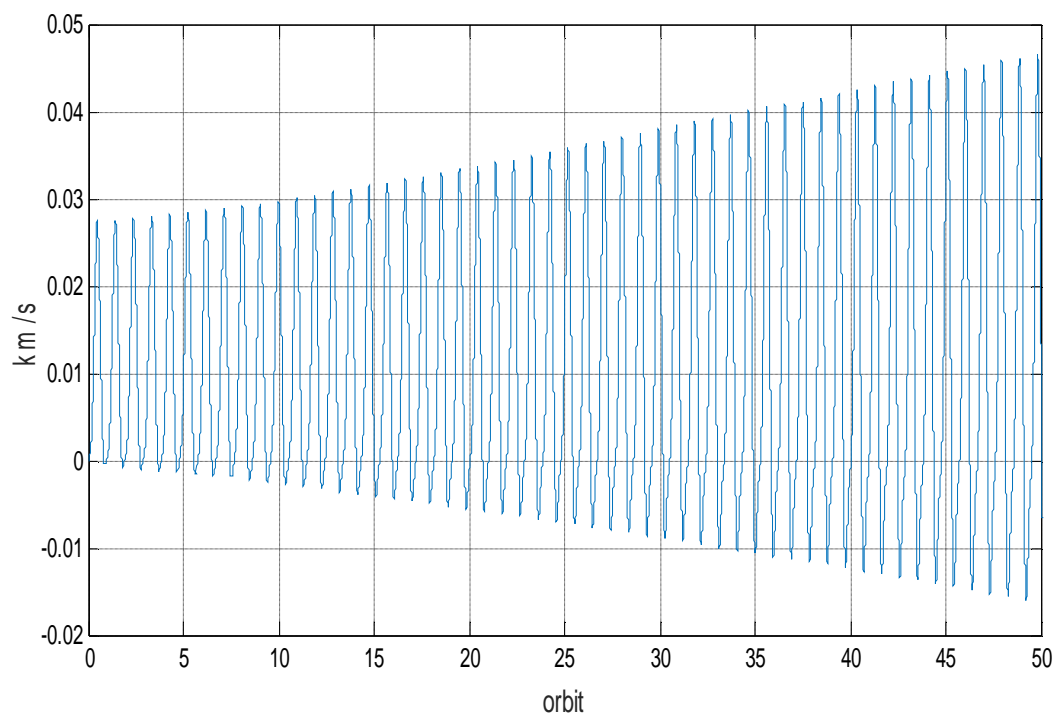

Figure 4, the magnitude of error in orbit velocity magnitude 


\section{CONTROL ALGORITHMS}

Generally, to apply feedback control to the orbit of the satellite, the actual position, and velocity of the satellite are compared to the reference values. Then the desired value and direction of thrust ac, are calculated based on the chosen control law, this thrust should force the actual position and velocity to follow the reference values and compensate the effect of the disturbance, ad. The communally used control laws are described below.

\subsection{PD controller}

PD control is the earliest and most commonly used control technique in the field of spacecraft attitude and orbit control; this is because, PD control has many advantages, such as simple structure, good stability, reliable, and easy tuning controller.

The family satellites swarm control algorithm computes the required control effort, $\mathrm{u}$, based on system dynamics model in (9)\&( 11), in order to track the reference orbital parameters $\left(r_{r \bullet f}, v_{r \bullet f}\right)$ specified by the following guidance algorithm

$$
u=K_{p} \Delta r+K_{d} \Delta v
$$

Then, the required control thrust, $a c$, will be calculated from the above-calculated control effort, 国, based on (14) as follows

$$
a c=-a g_{\text {ref }}+a g-u
$$

The proportional and derivative of PD control gains, $K_{p}$ and $K_{d}$ are determined based on the natural frequency $\omega_{n}$ and the damping ratio $\zeta$ of the desired response as follows

$$
k_{p}=\omega_{n}^{2} \& k_{d}=2 \zeta \omega_{n}
$$

\subsection{Linear quadratic regulator controller}

Linear quadratic regulator $(\mathrm{LQR})$ is a real-time closed-loop optimal control technique. Traditionally, LQR has been used on satellite orbit control because of its reliability and robustness. The LQR strategy is based on finding the optimal control effort, $\$$, which forces the $\Delta r \& \Delta v$ defined in the linear system (12),(13), (14) and to be minimum under the constraint of minimizing the following performance index $l$.

$$
\begin{gathered}
I=\frac{1}{2} \int\left(x(t)^{T} \cdot Q \cdot x(t)\right) \\
+\left(u(t)^{T} \cdot R \cdot u(t)\right)
\end{gathered}
$$

Where $\mathrm{Q}$ and $\mathrm{R}$ are the performance index weight matrices, $\mathrm{Q}$ is $\mathbf{n} \times \mathbf{I}$-dimensional, positive semi-definite state weighting matrix, and $\mathrm{R}$ is $\mathbf{r} \times \mathbf{0}$-dimensional positive definite control weight matrix. The term $x^{2} \cdot Q \cdot x$ in. خطأ! لم يتم العثور على مصدر المرجع measures the control accuracy, while, the term $\mathbf{u}^{T} \cdot R \cdot$ 国 measures the control effort.

According to the principle of minimization, the control effort $\mathrm{u}$ will be optimal when

$$
\begin{aligned}
& u(t)=-K(t) x(t) \\
& K(t)=R^{-1} \cdot B(t) \cdot P(t)
\end{aligned}
$$

Where $P(t)$ is a symmetric nonnegative definite matrix, and satisfy the Riccati matrix differential equation defined below 


$$
\dot{P}(t)=P(t) B(t) R^{-1} \cdot B(t) \cdot P(t) Q-P(t) A(t)-A^{T}(t) P(t)
$$

The state weighting matrix $\mathrm{Q}$ effects on the system dynamic tracking error, while the control weighting matrix $\mathrm{R}$ effects on the required control energy, consequently changing $\mathrm{Q}$ and $\mathrm{R}$ will change the $\mathrm{K}$ matrix, this matrix defines the control performance, thus it is required to choose the matrices $\mathrm{Q}$ and $\mathrm{R}$ carefully. The process of choosing $\mathrm{Q}$ and $\mathrm{R}$ will be done through many simulations with different values of $\mathrm{Q}$ and $\mathrm{R}$ while comprehensively considerations for fuel consumption and tracking errors are taken.Then the used control thrust will be calculated using ( 16)

\subsection{Sliding mode controller}

Sliding mode variable structure control is commonly used as a robust control technique, which has been successfully applied in the satellite orbit and attitude control field. The advantages of this technique are; strong robustness against the system parameters, and disturbances uncertainty. The design process of the sliding mode controller consists of two steps Choosing a suitable sliding manifold.

- Designing a control law.

Designing the sliding mode control to maintain the orbit of family satellite swarm members will be implanted into the system (12),(13), (14) following the above two steps as follows.

1. Choosing the sliding manifold.

Let the sliding manifold $S$ be

$$
S=\Delta r+\Delta v
$$

The sliding manifold is the subspace of the system state space, where the sliding variable equals zero

2. Designing a control law

$$
S=\{\Delta r, \Delta v: S=\mathbf{0}\}
$$

It is required to find control effort which drives the states of the system from a point in the state space to the sliding manifold $S=\mathbf{0}$ and keep it along sliding manifold, which is the stability problem of the sliding mode, and to show this, consider a Lyapunov candidate function ${ }^{3}$

$$
\begin{aligned}
& V=\frac{1}{2} S^{T} \cdot S \\
& \dot{V}=S^{T} \cdot S
\end{aligned}
$$

Subtitling ( 9),( 11),(14)and ( 22) in ( 25)

$$
\begin{aligned}
& \dot{V}=S^{T} \cdot(\Delta r+\Delta v) \\
& \dot{V}=S^{T} \cdot(\Delta v+u)
\end{aligned}
$$

And in order to be sure that derivative of Lyapunov candidate function $\mathrm{V}$ is negative (i.e. $\dot{V}<0$ ) the control effort ${ }^{3}$ will be choosing to be

$$
\begin{aligned}
& u=-K_{2} S-K_{2} \operatorname{sign}(S)-\Delta v \\
& \therefore v=S^{T} \cdot\left(-K_{1} S-K_{2} \operatorname{sign}(S)\right) \\
& b=-K_{1} S^{T} \cdot S-K_{2}|S|<0
\end{aligned}
$$


For the purpose of reducing of chattering effect, a common practice is to replace the term sign $(5)$ function in (28) by the following continuous saturation function

$$
\begin{aligned}
& \operatorname{sign}\left(S_{i}\right)=\left\{\begin{array}{c}
\frac{S_{i}}{\varepsilon} i f\left|S_{i}\right| \leq \varepsilon \\
\operatorname{sign}\left(S_{i}\right) i f\left|S_{i}\right|>\varepsilon \\
{[}
\end{array}\right. \text {. } \\
& i=1,2,3
\end{aligned}
$$

Then the used control thrust will be calculated using ( 16)

\section{SIMULATION RESULTS}

In order to select the suitable control algorithms to maintain the orbit of family satellite swarm, the abovemotioned control techniques are implemented using the same initial conditions, then the behavior of all algorithms are compared using the comparison factor mentioned in section 3

The initial conditions are presented in Table 1

Table 1: Initial conditions

\begin{tabular}{|c|c|c|c|}
\hline Parameter & $\begin{array}{c}\text { Initial } \\
\text { reference } \\
\text { orbital } \\
\text { parameter }\end{array}$ & $\begin{array}{c}\text { Initial } \\
\text { actual } \\
\text { orbital } \\
\text { parameter }\end{array}$ & Error \\
\hline$r(\mathrm{~km})$ & {$\left[\begin{array}{c}6861.4 \\
-2 \\
1\end{array}\right]$} & {$\left[\begin{array}{c}6863.4 \\
-2 \\
1\end{array}\right]$} & {$\left[\begin{array}{c}2 \\
-2 \\
1\end{array}\right]$} \\
\hline$v\left(\frac{\mathrm{km}}{\mathrm{s}}\right)$ & {$\left[\begin{array}{c}0 \\
-0.9777 \\
07.55891\end{array}\right]$} & {$\left[\begin{array}{c}-0.050 \\
-0.9277 \\
7.55891\end{array}\right]$} & {$\left[\begin{array}{c}-0.05 \\
0.05 \\
-0.05\end{array}\right]$} \\
\hline
\end{tabular}

The simulation results for the above initial conditions are presented below 


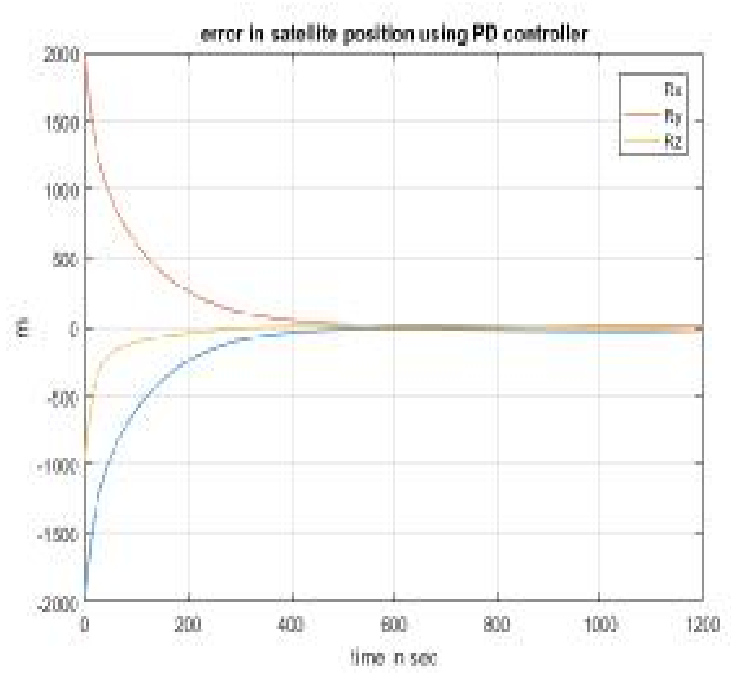

Figure 5, Error in satellite position using PD controller

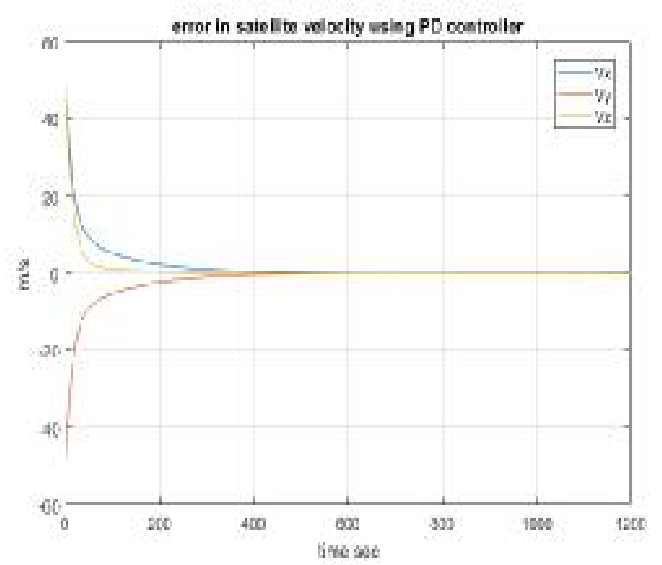

Figure 6, Error in satellite velocity using PD controller
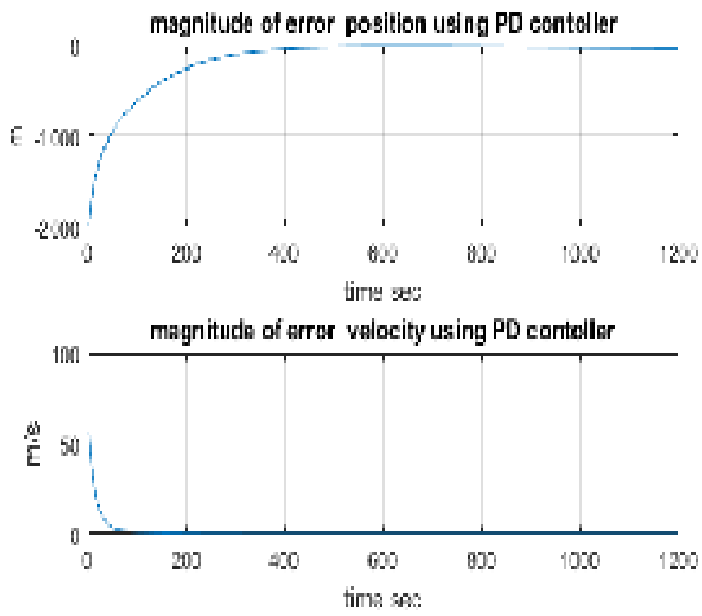

Figure 7, Magnetitude of error in position and velocity for the PD controller

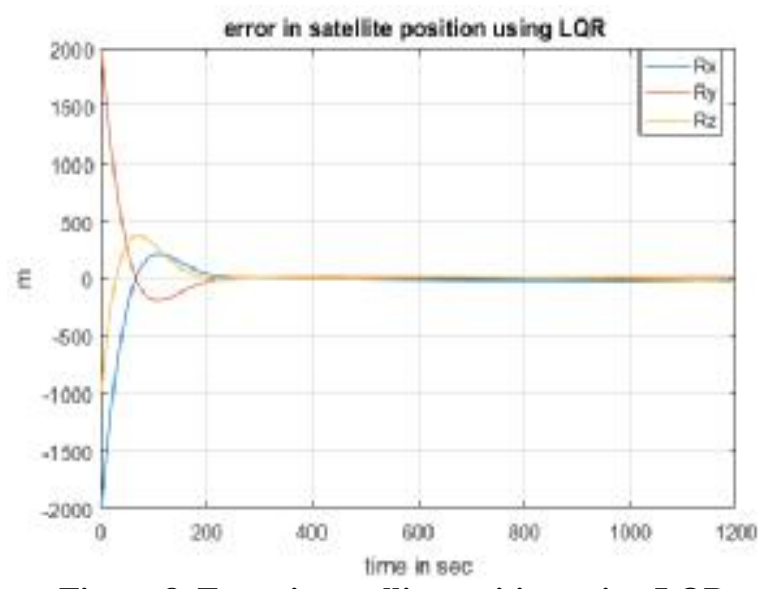

Figure 8, Error in satellite position using LQR

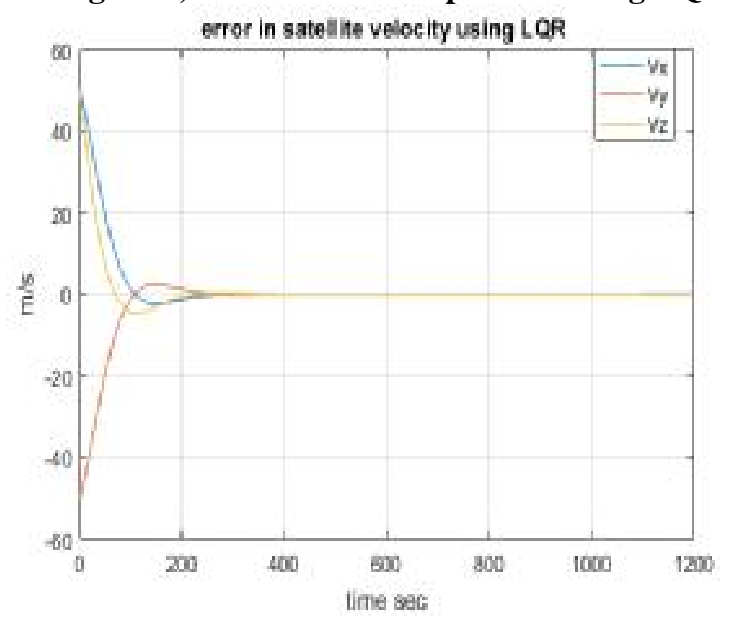

Figure 9, Error in satellite velocity using LQR 

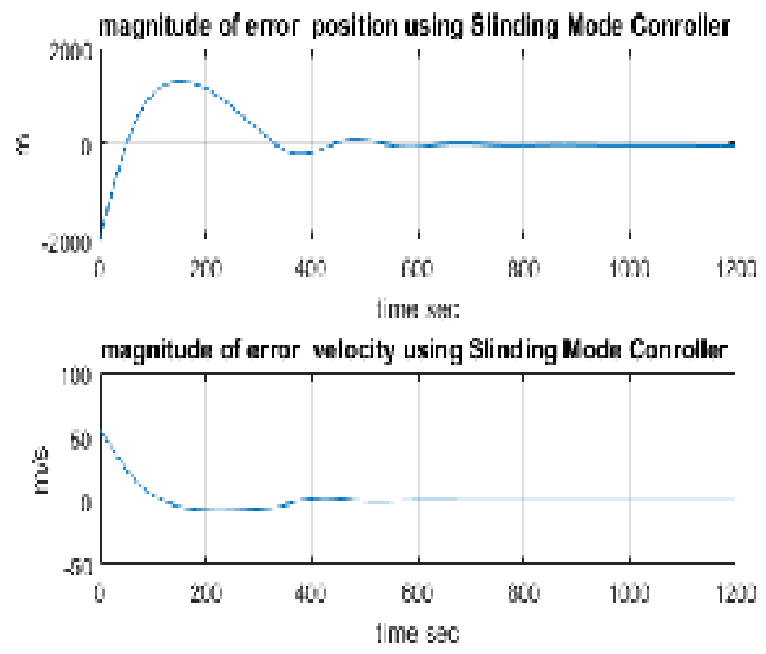

Figure 13, Magnetitude of error in position and velocity for Sliding mode controller
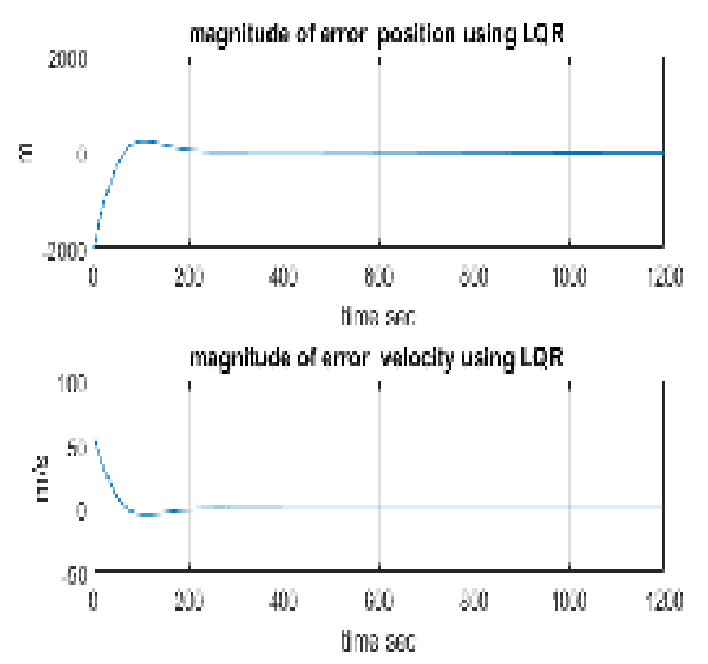

Figure 10, Magnetitude of error in position and velocity for $L Q R$

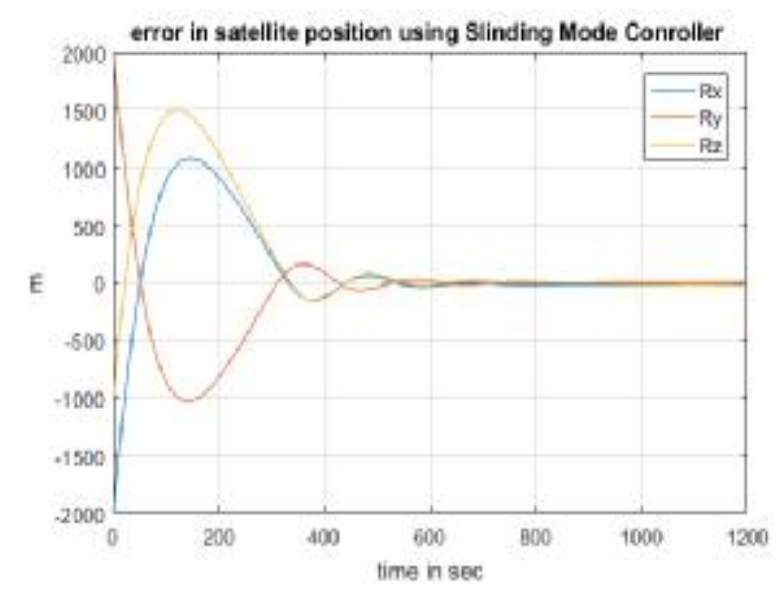

Figure 11, satellite position using Sliding mode controller

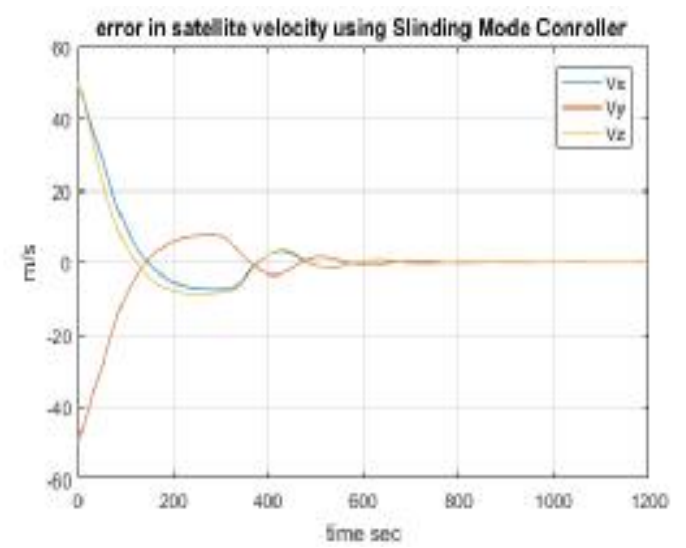

Figure 12, satellite velocity using Sliding mode controller 
Table 2 Compression between the performances of commonly used control techniques

\begin{tabular}{|c|c|c|c|}
\hline $\begin{array}{l}\text { Compression } \\
\text { factor }\end{array}$ & $\begin{array}{l}\text { PD } \\
\text { controller }\end{array}$ & LQR & $\begin{array}{l}\text { Sliding } \\
\text { mode }\end{array}$ \\
\hline $\begin{array}{l}\text { Required control } \\
\text { thrust for one } \\
\text { day }\left(\frac{m}{s^{2}}\right)\end{array}$ & 2.052 & 1.693 & 2.118 \\
\hline $\begin{array}{l}\text { Steady stat error } \\
\text { in } \quad \text { satellite } \\
\text { position }(\mathrm{km})\end{array}$ & 0.04 & 0.032 & 0.025 \\
\hline $\begin{array}{l}\text { Steady stat error } \\
\text { in } \\
\text { satellite } \\
\text { velocity }\left(\frac{m}{}\right)\end{array}$ & 0.1 & 0.08 & 0.06 \\
\hline Settling time $(s)$ & 301 & 169 & 420 \\
\hline $\begin{array}{l}\text { Execution time } \\
(s)\end{array}$ & $\begin{array}{l}4.1287 \mathrm{e}- \\
5\end{array}$ & $\begin{array}{l}5.1202 \mathrm{e}- \\
3\end{array}$ & $8.916 \mathrm{e}-4$ \\
\hline
\end{tabular}

\section{Dissection on simulation results}

As it is stated in the control concept section, the suitable control algorithm is the $\frac{m_{1}}{s}$ gorithm that achieves minimum control thrust, steady sat less than $0.15 \mathrm{~km}$ in position and $s$ in velocity, minimum settling time, and minimum execution time.

\section{The simulation results showed that}

1. The LQR needs minimum control effort

2. The thee control algorithms could achieve the mission requirements regarding the position and velocity error.

3. LQR is the fastest control algorithm (i.e. lowest settling time)

4. $\quad \mathrm{PD}$ is the lowest execution time and LQR is the highest execution time

Consequently, the decision is to use LQR because; it could achieve minimum control effort, steady-state error requirements, and minimum settling time even if it needs the highest execution time as this requirement has the lowest priority.

\section{CONCLUSION}

The problem of choosing the suitable orbit control algorithm of a satellite swarm for remote sensing application was addressed. In order to select the best control algorithm, compassion between PD, LQR, and sliding mode control algorithms was conducted, the traditional control performance parameters such as; minimum control effort, minimum steady stat error, minimum settling time and minimum execution time are used to select the best control algorithm. the simulation results showed that the LQR needs minimum control thrust, which is the most critical requirement, moreover, it achieves the required steady sate error in position and velocity, besides it is the lowest settling time, that is why it was selected for orbit control of the satellite swarm even it needs high execution time.

\section{REFERENCES}

1. Olsen, N., Friis-Christensen, E., Floberghagen, R., Alken, P., Beggan, C. D., Chulliat, A.,.Visser, P. N."The Swarm Satellite Constellation Application and Research Facility (SCARF) and Swarm data products. Earth", Planets and Space, 64(11),1189-1200. DOI: 10.5047/eps.2013.07.001,2013.

2. Jose van den Jssel, Joa oEncarnac $\mathrm{a}^{2}$ o, "Precise science orbits for the Swarm satellite constellation” Advances in Space Research, 2015 
3. Engelen, S., Verhoeven, C., and Bentum, M. "OLFAR, a radio telescop e based on nanosatellites in moon orbit" 24th Annual American Institute of Aeronautics and Astronautics/Utah State Univ. Conference on Small Satellites, Logan, UT, 2010

4. K. Alfriend, H. Schaub, Dynamics, and control of spacecraft formations: Challenges and some solutions, Journal of Astronautical Sciences 48 (2) (2000) 249-267.

5. D.P. Scharf, F.Y. Hadaegh, S.R. Ploen, A survey of spacecraft formation flying guidance and control (part II): Control, in Proceedings of the 2004 American Control Conference, Boston, MA, June 30-July 2, 2004.

6. Ahmad Farrag, Tarek Mahmoud, Ahmed Elrafie," New Conceptual Design of Satellites Swarm For Remote Sensing Application", Journal of aerospace technology and management, 2019.

7. B. S. Lee, Y. Hwang, H. Y. Kim, S. Park, East-West station-keeping maneuver strategy for CMOS satellite using iterative process. Advances in space research, vol. 47, pp.149$159,2011$.

8. Y. N. Yang, J.Wu, W. Zheng, Station-keeping control for a stratospheric airship platform via fuzzy adaptive backstepping approach. Advances in Space Research, vol. 51, pp.1157$1167,2013$.

9. Königsmann, H. J., Collins, J. T., Dawson, S., and Wertz, J. R. "Autonomous orbit maintenance system." ActaAstronaut., 39(9-12), 977-985,1996

10. S. A. Parvez and G. Q. Xing, "Autonomous orbit control with position and velocity feedback using modern control theory," U.S. Patent 6,089,507 2000;

11. Wertz, J. R., and Gurevich, G. "Applications of autonomous onboard orbit control." Proc., 11th AAS/AIAA Space Flight Mechanics Meeting, Santa Barbara, CA. 2001

12. Garulli, A., Giannitrapani, A., Leomanni, M., and Scortecci "Autonomous low-Earthorbit station-keeping with electric propulsion." Journal of Guidance and Control Dynamics, 2011.

13. Zhang, Z., Ling, W.Y.L., Tang, H. et al. "A review of the characterization and optimization of ablative pulsed plasma thrusters " Rev. Mod. Plasma Phys. 3: 5. https://doi.org/10.1007/s41614-019-0027-z

14. F. Liu et al.," Guidance and Control Technology of Spacecraft on Elliptical Orbit, Navigation: Science and Technology", Doi:10.1007/978-981-10-7959-7_8 , Springer Nature Singapore Pte Ltd. and National Defense Industry Press, 2019

15. LU Shan. "A Study on Relative Orbital Dynamics and Control of Spacecraft Autonomous Rendezvous ". Beijing: Beihang University, 2009.

16. LU Shan, XIA Yong-Jiang. "Robust Sliding Mode Control for Autonomous Rendezvous of Spacecraft on Elliptical Orbit. Aerospace Shanghai, 2012"

17. Li Jian, Wang Dongqing, Wang Limei. "Design of Fuzzy PID Controller and its Simulation Based on MATLAB [J]. Industrial Control Computer", 2011.

18. Enrico Canuto, Carlo Novara, Luca Massotti "Spacecraft dynamics and control - the embedded model control approach" Elsevier, 2018.

19. Marcel J. Sidi., "Spacecraft Dynamics and Control." Cambridge University Press, 2000.

20. Mohamed Okasha, Brett Newman," Relative Motion Guidance, Navigation and Control for Autonomous Orbital Rendezvous" Journal of Aerospace Technology and Management, 2014.

21. Utkin, V.I. “Sliding Modes in Control Optimization.”, Springer-Verlag, 1992.

22. Slotine, J. E. and W. Li. “ Applied Nonlinear Control”, Prentice-Hall, 1991.

23. Bruce A. Conway, "Spacecraft Trajectory Optimization", 